\title{
Ciclo económico y prima por riesgo en el mercado accionario colombiano
}

Business Cycle and Risk Premium in the Colombian Stock Market

Andrés Mauricio Gómez Sánchez*

José Gabriel Astaiza Gómez**

Recibido: 01/04/2013

Aprobado: 04/06/2013

Magíster en Economía Aplicada. Docente titular en el Departamento de Economía, Universidad del Cauca, Popayán, Colombia; miembro de los grupos de investigación Gicea y Entropía. [amgomez@unicauca.edu.co].

** Especialista en Finanzas. Catedrático, Universidad del Cauca, Facultad de Ciencias Contables, Económicas y Administrativas. [jgastaiza@unicauca.edu.co]. 


\title{
Resumen
}

Este artículo presenta una revisión de la relación entre la prima por riesgo ex post del mercado accionario y los ciclos económicos observados en Colombia recurriendo a la metodología Hodrick-Prescott. Con información trimestral desde el cuarto trimestre de 2001 al tercer trimestre de 2012, la evidencia estadística muestra que los aumentos y disminuciones de la prima por riesgo ex post siguen un comportamiento contracíclico en sintonía con las investigaciones realizadas hasta ahora para Estados Unidos y economías emergentes, aunque con relaciones no contemporáneas con el consumo privado. Adicionalmente se encuentra que en la última década la prima por riesgo en Colombia sigue un proceso de modelos autorregresivos de media móvil (ARMA, por las siglas en inglés de AutoRegressive Moving Average Models), mostrando que no varía por lo menos en dos trimestres contiguos y cuyo comportamiento es generado en parte por eventos externos al nivel de actividad económica nacional acaecidos en periodos pasados cercanos.

\section{Palabras clave}

Ciclo económico, consumo, PIB, tasa de desempleo, prima por riesgo.

\begin{abstract}
Through the Hodrick-Prescott methodology this paper presents a review about the relationship between the ex post risk premium of the stock market and business cycles observed in Colombia. Through quarterly information from the fourth quarter of 2001 to the third quarter of 2012, statistical evidence shows that the increase and decrease of ex post risk premium follow a countercyclical behavior in tune with existing research conducted about the United States and emerging economies, although with non-contemporary relationships with private consumption. In addition, it is found that in the last decade the Colombian risk premium follows a process of Auto Regressive Moving Average Models (ARMA), showing that there is no variation in at least two consecutive quarters and whose behavior is generated in part by external events at the domestic economic activity level experienced in near past periods.
\end{abstract}

\section{Key Words}

Business cycle, consumption, GDP, unemployment rate, risk premium.

Clasificación JEL: D91, E21, E32, E44, G11, G12, G14 


\section{Introducción}

El concepto de prima por riesgo ha estado desde tiempos tan tempranos en la literatura económica como la publicación de la obra Principles of Political Economy, de John Stuart Mill ${ }^{1}$ en 1848 (Ibbotson y Goetzmann, 2005), aunque no fue sino hasta la formulación del Capital Asset Pricing Model (CAPM), a su vez basado en el modelo de comportamiento del inversionista averso al riesgo de Markowitz (1952), que la prima por riesgo comenzó a ser un elemento fundamental como información, no solo para la composición de portafolios en los mercados bursátiles, sino también para la valoración de empresas y la evaluación de nuevas inversiones en activos reales. ${ }^{2}$ Ya por varias décadas la prima por riesgo ha llamado la atención de economistas, así como de quienes se desempeñan profesionalmente en el sistema financiero, dando lugar a al menos 320 artículos publicados sobre el tema (Siegel, 2005) y 100 libros publicados entre 1979 y 2008 con recomendaciones sobre su estimación (Fernández, 2009).

Entre los determinantes de la prima por riesgo se pueden encontrar la información en los mercados, la aversión al riesgo, la liquidez del mercado y el riesgo económico, entre otros (Damodaran, 2012). Este último está asociado a los cambios en el nivel de actividad económica reflejado en variables como el producto interno bruto, el consumo privado y las tasas de desempleo, lo cual afecta la percepción del riesgo entre los agentes. En sí mismo, el ciclo económico, o más bien, el reconocimiento de la existencia permanente de fluctuaciones en la actividad económica a través del tiempo, ha sido tema de investigación intensa por lo menos desde la segunda mitad del siglo XIX, implicando a un cambio en la estructura teórica estática del equilibrio económico de la escuela clásica y marginalista hacia la teoría económica dinámica (Kuznets, 1930). Aunque en sus inicios la estimación del ciclo económico tuvo su enfoque en los comovimientos de series desagregadas entre sectores de la economía, actualmente gran parte del análisis se realiza mediante series individuales, lo que se hace evidente en trabajos como los de Hess e Iwata (1997) y la ampliamente aceptada metodología de Hodrick y Prescott (1997).

1 En su obra Mill (1848) expresa que un granjero podría estar dispuesto a realizar un gasto de capital en su tierra si dicha inversión le brindara un exceso de rendimiento por encima del interés que habría de pagar por un préstamo y el valor del riesgo.

2 En el CAPM la prima por riesgo es medida como el rendimiento esperado del conjunto de activos que componen el mercado accionario menos la tasa libre de riesgo (Fama y French, 2004, p. 29). 
La relación entre el ciclo económico y la prima por riesgo ha sido documentada en trabajos como los de Lustig y Verdelhan (2012), Lettau, Ludvigson y Wachter (2008), Salomons y Grootveld (2003), Ferson y Harvey (1991) y Fama y French (1989), entre otros. Sin embargo, la investigación acerca de dicha relación es escasa en Colombia, no habiendo encontrado referentes en el rastreo bibliográfico realizado. En ese sentido, el presente documento se suma a la investigación sobre el tema, abordándola mediante la observación del ciclo económico y la prima por riesgo ex post ${ }^{3}$ colombianos.

El contenido del artículo se divide en ocho secciones, incluyendo la introducción. En la segunda y tercera secciones se presenta un acercamiento teórico de la relación entre la actividad económica y la prima por riesgo, así como investigaciones empíricas realizadas sobre el tema. En la cuarta y quinta secciones se describe la información utilizada y la metodología de estimación de los ciclos. En las secciones sexta y séptima se expone la relación encontrada entre la prima por riesgo y los ciclos en Colombia, incluyendo un análisis econométrico. Por último, se muestran las conclusiones.

\title{
2. Marco teórico
}

En la teoría de valoración de activos el inversionista realiza una elección óptima cuando el valor esperado de su utilidad marginal derivado del incremento de su consumo futuro se iguala a la pérdida de utilidad marginal que afronta dicho inversionista al sacrificar su consumo presente y comprar un activo. En palabras de Mehera:

\begin{abstract}
El deus ex machina en la teoría de valoración de activos es que los activos son valorados de tal forma que, ex ante, la pérdida de utilidad marginal incurrida al sacrificar consumo presente y comprar un activo a un precio determinado, es igual a la ganancia esperada de utilidad marginal, que depende del incremento previsto en consumo cuando el activo brinda sus ganancias en el futuro (Mehra, 2003, p. 55)
\end{abstract}

Siendo la utilidad marginal del consumo decreciente, lo anterior implica que en niveles elevados de consumo se requieren tasas de ganancia esperadas más bajas para compensar la pérdida de utilidad en el presente. En niveles bajos de consumo, cuando la

3 En la literatura financiera existen claramente cuatro perspectivas diferentes de la prima por riesgo: la histórica o ex post, la esperada, la exigida y la implícita. Al respecto puede revisarse Fernández (2009). Como un referente de su utilización ex post o histórica, pueden verse Salomons y Grootveld (2003). 
utilidad marginal es mayor, se requieren tasas de retorno esperadas más elevadas para compensar la pérdida de utilidad marginal en el presente cuando se renuncia a cierto consumo para comprar el activo.

Teóricamente, Lucas (1978) mostró que en una economía en la cual los derechos de propiedad sobre las unidades productivas se determinan en un mercado de acciones competitivo y en la que cada acción da a su propietario el derecho sobre el producto, las decisiones del consumidor sobre su consumo presente y la composición de su portafolio futuro dependen, entre otras, de la información relevante que posee sobre el estado actual y futuro de la producción, o estados presente y futuro de la economía. De esa manera, con una aversión relativa al riesgo menor a la unidad, nueva información optimista sobre los dividendos futuros lleva a precios elevados de los activos.

\section{Antecedentes empíricos de los ciclos económicos y la prima por riesgo}

Desde el punto de vista empírico, Lustig y Verdelhan (2012) brindaron evidencia histórica para Estados Unidos de que la inversión en acciones durante las recesiones de la posguerra ofreció un exceso promedio de retorno del 11,3\%, superior al 5,3\% correspondiente a la inversión en épocas de expansión económica. Adicionalmente mostraron que la razón de Sharpe fue contracíclica entre 1945 y 2009. Lettau, Ludvigson y Wachter (2008) encontraron una fuerte correlación entre la volatilidad macroeconómica y los precios de las acciones en Estados Unidos utilizando información sobre el consumo personal per cápita y el producto interno bruto. Mediante un modelo de valoración de activos con agentes racionales y visión a futuro, concluyeron que la disminución en el riesgo macroeconómico implica una caída en la prima por riesgo y un alza en los precios de las acciones. Salomons y Grootveld (2003) expusieron evidencia estadística de que las variaciones de la prima por riesgo ex post de las economías emergentes se relacionan más con el ciclo económico global que con cambios estructurales derivados de la liberación de los mercados de capitales en dichas economías. Ferson y Harvey (1991), utilizando información de la National Bureau of Economic Research sobre los ciclos económicos, un índice accionario ponderado por capitalización con títulos listados en la Bolsa de Nueva York y el retorno de los Treasury bill a un mes, observaron gráficamente que la prima por riesgo esperada se incrementó durante las contracciones económicas y alcanzó máximos cercanos a los valles del ciclo. Fama y French (1989) encontraron 
que en Estados Unidos el rendimiento por dividendos está correlacionado con el default spread, medido como la diferencia entre el rendimiento de un portafolio con bonos corporativos y el rendimiento de bonos Aaa. A su vez, el default spread es alto en periodos con una actividad económica precaria y bajo en periodos de fuerte actividad económica. Al respecto expresaron:

Un argumento para estos resultados es que cuando las condiciones económicas son precarias, el ingreso es bajo y los retornos esperados de los bonos y las acciones deben ser altos para inducir a la sustitución de consumo por inversión. Cuando los tiempos son buenos y el ingreso es alto, el mercado se vacía a niveles más bajos de rendimientos esperados (Fama y French, 1989, p. 48).

Lo anterior es consistente con la noción de que cuando la actividad económica está en expansión los niveles de consumo son elevados y la utilidad marginal es baja, entonces la prima por riesgo debe también ser baja. En el caso opuesto, cuando la economía se contrae, los niveles de consumo son bajos y la utilidad marginal del consumo es alta, la prima por riesgo debe ser elevada. En ese orden de ideas el presente artículo ofrece una revisión de la relación entre la prima por riesgo observada y la actividad económica en Colombia.

\section{Información}

Como variables de referencia sobre las condiciones macroeconómicas en Colombia se utilizan las series trimestrales desestacionalizadas del producto interno bruto a precios constantes de 2005 , el consumo privado y las tasas de desempleo correspondientes a los meses de marzo, junio, septiembre y diciembre desde el cuarto trimestre de 2001 al tercer trimestre de 2012 de acuerdo con la disponibilidad de información del Departamento Administrativo Nacional de Estadística. ${ }^{4}$

Con respecto al comportamiento del mercado de acciones, se considera que el índice utilizado debe ser ponderado por capitalización y haber incorporado empresas que en su momento cotizaron sus acciones pero luego quebraron o fueron compradas. Lo an-

$4 \quad$ Pueden descargarse las series de la página del DANE (www.dane.gov.co). En la construcción de una serie que refleje los ciclos económicos en Colombia, resultaría interesante revisar en estudios posteriores además del desempleo, el PIB y sus componentes, el indicador de consumo de energía eléctrica. 
terior debido a que por una parte, los retornos del mercado están más inclinados hacia acciones con mayor capitalización, y por otra, calcular los rendimientos únicamente de empresas maduras o de largo recorrido daría retornos especialmente altos (Damodaran, 2012, p. 24). En ese sentido, el Índice General de la Bolsa de Valores de Colombia (IGBC) como índice ponderado por capitalización es apropiado para estimar los retornos del mercado de acciones colombiano. ${ }^{5}$ Se utilizan así tasas de retorno trimestrales calculadas con los valores del IGBC del último día transado en los meses de marzo, junio, septiembre y diciembre, iniciando el 28 de septiembre de 2001 y finalizando el 28 de septiembre de $2012 .^{6}$

Para la estimación de la tasa libre de riesgo idealmente se requeriría la tasa de rendimiento de bonos del Gobierno cupón cero, a un plazo coincidente con el horizonte de inversión. Lo anterior garantizaría que el activo en cuestión cumpla con dos requisitos fundamentales para ser considerado libre de riesgo. Primero, que no tenga riesgo de impago, y segundo, que su retorno presente sea igual a su rendimiento esperado (Damodaran, 2008, p. 6). En esos términos el rendimiento a vencimiento de los TES cupón cero con vencimiento a septiembre de 2014 podría desempeñarse de manera aceptable pero la información es notoriamente limitada para los periodos previos al tercer trimestre de 2006, contando con algunos días bursátiles sin registro en los periodos posteriores. ${ }^{7}$ Por tal motivo y siguiendo la usanza del sistema financiero e investigación en Colombia, se recurre a las tasas mínimas de expansión del Banco de la República como tasa libre de riesgo; ${ }^{8}$ estas corresponden a las observaciones de los meses de marzo, junio, septiembre y diciembre de cada año, ${ }^{9}$ expresadas en forma trimestral.

5 Para investigaciones posteriores sería importante considerar la construcción de un indicador más ajustado a las características deseables con las series históricas de acciones representativas del mercado colombiano.

6 Los valores mensuales del IGBC pueden ser descargados de la página del Banco de la República (www. banrep.gov.co).

7 También, los TES 2020 podrían ser adecuados debido a que son más líquidos y su vencimiento es posterior al periodo de estudio, pero su fecha de emisión es posterior a la fecha de inicio de las demás series utilizadas y cuentan con una tasa cupón del 11\%, lo que implica que su rendimiento a vencimiento no es libre de riesgo porque existe el riesgo de reinversión.

8 Entre otras publicaciones que recurren a la tasa mínima de expansión como medida de la tasa libre de riesgo en Colombia se pueden encontrar García y Moreno (2011); Martínez y Murcia (2007) y Jara (2006).

9 Pueden ser descargadas de la página del Banco de la República (www.banrep.gov.co). 
Ciclo económico y prima por riesgo en el mercado accionario colombiano

ANDRÉS MAURICIO GÓMEZ SÁNCHEZ

JOSÉ GABRIEL ASTAIZA GÓMEZ

En esos términos, la prima por riesgo observada (desde ahora ERP) es igual a la diferencia entre la tasa de rendimiento del IGBC y la tasa mínima de expansión del Banco de la República.

\section{Estimación de los ciclos}

Para capturar los escenarios de fuerte y débil actividad económica se observan los ciclos del PIB, el consumo privado y la tasa de desempleo, y se obtienen mediante el filtro de Hodrick-Prescott. El marco conceptual de dicho filtro consiste en que una serie es la suma de su crecimiento tendencial y su componente cíclico de donde los valores por encima o por debajo del componente tendencial son:

$$
c_{t}=y_{t}-g_{t}
$$

y su promedio debe ser igual a cero en largos periodos de tiempo. Así, el crecimiento tendencial de la serie se encuentra resolviendo (Hodrick y Prescott, 1997, p. 3):

$$
\operatorname{Min}_{\left\{g_{t}\right\}_{t=-1}^{T}}\left\{\sum_{t=1}^{T} c_{t}^{2}+\lambda \sum_{t=1}^{T}\left[\left(g_{t}-g_{t-1}\right)-\left(g_{t-1}-g_{t-2}\right)\right]^{2}\right\}
$$

donde $\lambda$ es el parámetro que penaliza las desviaciones alrededor del componente tendencial y, por tanto, cuanto más elevado, más “suave" es la serie encontrada. ${ }^{10}$ De esa forma, periodos en los cuales la diferencia entre el valor observado del PIB (o el consumo privado) y su nivel tendencial es mayor a cero (), corresponden a tiempos de altos niveles de actividad económica (expansiones) y periodos en los que dicha diferencia es menor a cero () reflejan épocas de baja actividad económica (recesiones). Lo contrario sucede con la tasa de desempleo.

10 Para información trimestral del mercado estadounidense, Hodrick y Prescott (1997, p. 4) consideran que un valor adecuado para $\lambda$ es 1600 . 


\section{Relaciones cíclicas de la prima de riesgo ${ }^{11}$}

Al revisar la relación entre el PIB y la prima por riesgo (ERP) se muestra que la correspondencia es contracíclica, ya que el coeficiente de correlación es negativo (-0.28). Esto indica que las expansiones de la economía están asociadas a contracciones en la ERP; por lo tanto, cuando la economía colombiana experimenta altos niveles de actividad económica, la prima por riesgo experimenta bajos niveles. La figura 1 muestra este resultado.

Figura 1.

Ciclos PIB y prima por riesgo. Datos normalizados, 2001:4-2012:3

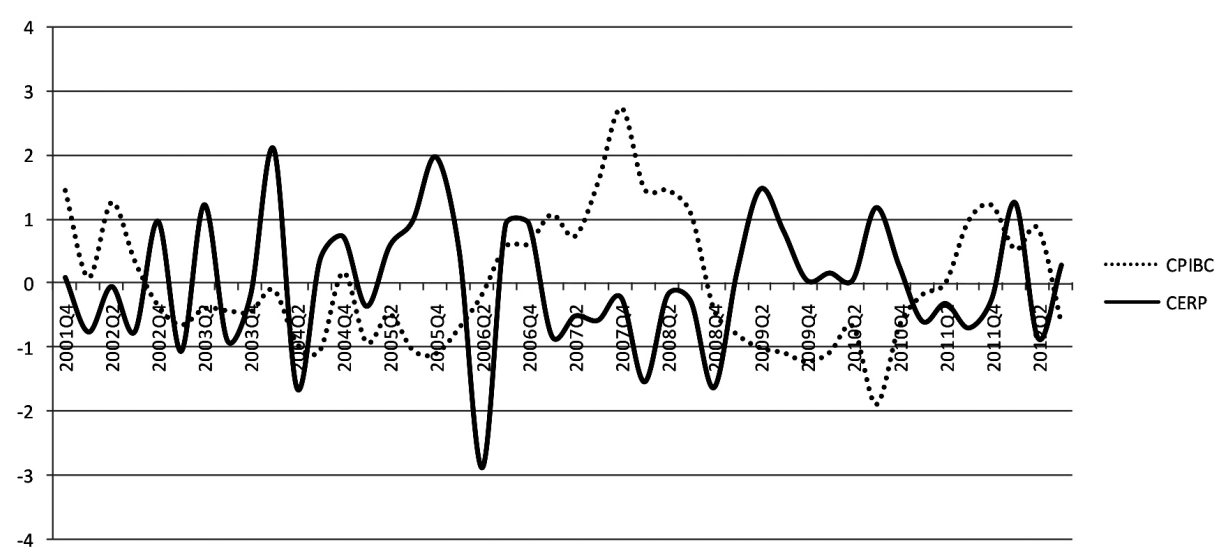

CPIBC: ciclo PIB Colombia; CERP: ciclo prima por riesgo. Las unidades de medida están expresadas en desviaciones estándar.

Al comparar la ERP con el desempleo se encuentra que la relación es procíclica, pues el coeficiente de correlación es positivo (0.11), de tal forma que épocas de altas primas por riesgo se compaginan con altos niveles de desempleo, y viceversa. Finalmente, la relación entre la ERP y el consumo privado evidencia una relación contracíclica (-0.34); mostrándose que los altos consumos de los hogares siempre están acordes con bajos niveles de primas por riesgo (ver figura 2).

11 Las series fueron normalizadas para poder ser comparadas bajo una misma medida, ya que en niveles las cifras son muy disimiles, por ejemplo la ERP o el desempleo están capturados en porcentaje y el PIB en millones de dólares. Bajo la normalización ahora están expresadas en desviaciones estándar. 
Ciclo económico y prima por riesgo en el mercado accionario colombiano

ANDRÉS MAURICIO GÓMEZ SÁNCHEZ

JOSÉ GABRIEL ASTAIZA GÓMEZ

\section{Figura 2.}

Ciclos prima por riesgo, consumo de los hogares y tasa de desempleo.

Datos normalizados, 2001:4-2012:3

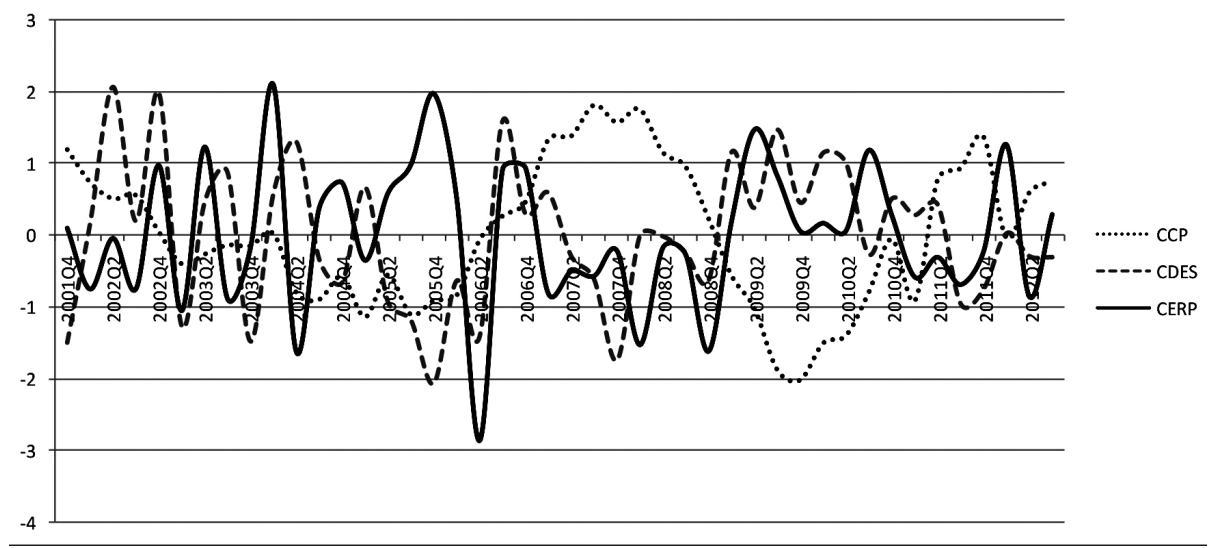

CCP: ciclo consumo privado; CDES: ciclo del desempleo; CERP: ciclo de la prima por riesgo. Las unidades de medida están expresadas en desviaciones estándar.

\section{Modelo econométrico}

De acuerdo con los resultados hallados sobre los comportamientos cíclicos, se plantea el siguiente modelo econométrico:

$$
C E R P_{t}=\beta_{1}+\beta_{2} C C P_{t}+\beta_{3} C D E S_{t}+\beta_{4} C P I B C_{t}+\beta_{5} A R(1)_{t}+\beta_{6} M A(1)+U_{t}
$$

donde $C E R P^{12}$ describe el ciclo de la prima por riesgo observada, CCP denota el ciclo consumo privado y CDES el ciclo del desempleo. La variable autorregresiva AR (1) se incluye para evitar posibles problemas de autocorrelación y por otro lado plantear la posibilidad de que la prima por riesgo en periodos tan cortos (trimestres) no pueda cambiar de forma muy rápida, por lo que muy posiblemente esta dependa hoy de lo que sucedió en el trimestre anterior. Debido a que las variables bursátiles presentan comportamientos muy volátiles sobre todo con fenómenos exógenos no previstos, se in-

2 Se recurre al ciclo de la ERP para ser consistentes con los ciclos de las demás variables y poder interpretar de forma correcta los parámetros. Aunque no se muestra en el documento, es importante notar que la ERP y su ciclo (CERP) son esencialmente iguales debido a que la primera no cuenta con una tendencia a través del tiempo ya que es una tasa de crecimiento. 
cluye un promedio móvil MA (1). Finalmente, Ut son los términos aleatorios de error que se supone son bien comportados, es decir, presentan una media igual a cero, varianza constante y covarianzas iguales a cero. ${ }^{13}$

Debe tenerse en cuenta que los comportamientos cíclicos de cualquier variable por definición son estacionarios, es decir, no tienen comportamientos erráticos con promedios cambiantes o altas varianzas, lo cual implica que no debe hacerse un tratamiento especial (como diferenciar la serie) para que estén bien comportadas, por tanto se dice en el lenguaje tradicional que el orden de integración de la serie es nulo, I(0). En este orden de ideas no se requiere hacer un análisis de raíces unitarias o de cointegración con las cifras utilizadas, lo que garantiza que los resultados de la regresión planteada en la ecuación 1 sean no espurios o falsos.

Se espera que la relación entre el ciclo del consumo privado y la prima por riesgo sea negativa, ya que cuando se presentan expansiones en la economía el consumo es elevado, lo que permite requerir un menor rendimiento. Por el lado del desempleo la relación deberá ser positiva, pues un desempleo elevado implica que en general los agentes requieran tasas de retorno más elevadas para sustituir consumo por inversión, por lo que la prima por riesgo aumenta. Con respecto al PIB, las recesiones deberían generar un mayor rendimiento requerido y las expansiones uno menor, por lo cual se espera también una relación inversa. Frente a la variable autorregresiva AR (1) su signo no es claro de forma a priori, ya que los cambios en la prima por riesgo podrían trasladarse al periodo siguiente o no, dependiendo de la coyuntura. Lo que sí debe plantearse sin temor a equivocaciones es que su valor debe ser inferior a la unidad, pues como se advirtió anteriormente, las series son integradas de orden cero. Finalmente, en lo concerniente al proceso MA (1), su carácter aleatorio impide conocer ex ante su signo.

En este orden de consideraciones, para lograr el mejor ajuste posible se exploraron varias formas funcionales (FF) a partir del modelo inicial. Los resultados se muestran de forma general en la tabla $1 .^{14}$

13 Se advierte que la relación causal del modelo no necesariamente puede llegar a ser contemporánea, o sea que el riesgo actual puede estar influenciado por comportamientos pasados de las variables consideradas.

14 Hay que considerar que las variables cíclicas están presentadas de forma normalizada, de tal forma que los valores numéricos expuestos deben tomarse con precaución. Ellos están denotando desviaciones estándar y no valores en niveles (es decir, valores sin ningún tipo de tratamiento estadístico), por lo que 
Ciclo económico y prima por riesgo en el mercado accionario colombiano

ANDRÉS MAURICIO GÓMEZ SÁNCHEZ

JOSÉ GABRIEL ASTAIZA GÓMEZ

Tabla 1.

Estimaciones del modelo

\begin{tabular}{lcccccc}
\hline & FF1 & FF2 & FF3 & FF4 & FF5 & FF6 \\
\hline C & $(-0.01)^{* * *}$ & $(-0.01)^{* * *}$ & $(-0.00)^{* * *}$ & & $(-0.01)^{* * *}$ & \\
CCP & $(-0.18)^{* * *}$ & & & & & $(-0.02)^{* *}$ \\
CCP(-1) & & $(-0.37)^{*}$ & $(-0.04)^{*}$ & $(-0.05)^{*}$ & $(-0.04)^{*}$ & \\
CDES & $(-0.03)^{* * *}$ & $(0.06)^{*}$ & $(0.01)^{* *}$ & $(0.008)^{*}$ & & \\
CDES(-1) & & & & & $(-0.009)^{* *}$ & $(-0.005)^{* * *}$ \\
CPIBC & $(-0.18)^{* * *}$ & & & & & \\
CPIBC(-1) & & & & & & $(-0.03)^{*}$ \\
AR(1) & $(-0.24)^{* * *}$ & $(0.29)^{* * *}$ & $(-0.60)^{*}$ & $(0.33)^{* *}$ & $(-0.65)^{*}$ & $(0.17)^{* * *}$ \\
MA(1) & $(-0.96)^{*}$ & $(-1.32)^{*}$ & & $(1.27)^{* *}$ & & $(-0.96)^{*}$ \\
MA(2) & & & $(-0.91)^{*}$ & & $(-0.90)^{*}$ & \\
R cuadrado & 0,42 & 0,62 & 0,50 & 0,59 & 0,49 & 0,46 \\
p-Valor F & 0,00 & 0,00 & 0,00 & & 0,00 & \\
\hline
\end{tabular}

$* p<5 \%, * * p<10 \%, * * * p>10 \%$; donde FF es forma funcional.

La estimación del modelo inicial (FF1) muestra un ajuste muy bajo y con signos algo contradictorios. De las restantes formas funcionales exploradas, el modelo 4 es el que mejor bondad estadística muestra a nivel global. ${ }^{15}$ En él no figura el comportamiento del PIB, ya que resultó estar relacionado linealmente con el consumo privado, ni tampoco el intercepto, pues la CERP, al estar capturada en desviaciones estándar (debido a la normalización) inicia muy cerca al valor de cero, como lo mostraron las figuras anteriores. Adicionalmente cumple con los signos esperados, la bondad de ajuste es alta, al igual que su significancia estadística. ${ }^{16}$

su interpretación económica no es procedente. Así, los coeficientes de la ecuación 1 deben evaluarse por los signos mostrados y por su significancia estadística, pero no por sus valores.

15 Ver anexo 1 para los resultados específicos de ambos modelos.

16 En este tipo de modelos, donde todas sus variables son cíclicas, esto es, que no se presentan tendencias en el tiempo y su comportamiento es accidentado o errático, los ajustes regularmente tiende a ser muy bajos, como lo muestra la tabla 1, por lo que obtener un ajuste del 59\% lo constituye en un modelo confiable. 
En este orden de ideas, la estimación evidencia que la relación entre el consumo privado y la prima por riesgo es inversa pero no contemporánea, es decir que si el consumo experimentó una expansión en el trimestre anterior, en el trimestre actual la prima por riesgo es baja y viceversa, ceteris paribus. Con el desempleo la correspondencia es directa y contemporánea, por tanto, en épocas de crecimiento económico la prima por riesgo ex post tiende a ser baja y en las recesiones tiende a ser alta, lo cual es consistente con la idea de que los agentes deben ser compensados con altas tasas de rendimiento cuando la actividad económica es negativa y su utilidad marginal alta, para inducirlos a sustituir consumo por inversión y viceversa.

Por el lado del proceso autorregresivo AR(1), se evidencia que la prima por riesgo no cambia de forma muy rápida de un trimestre a otro, es decir, no es muy fluctuante, por lo menos no para dos trimestres consecutivos. Finalmente, el proceso MA (1) muestra que la prima por riesgo depende de choques aleatorios externos de forma no contemporánea, de los cuales se desconoce su origen pero podrían provenir de causas exógenas a la economía como implementaciones de políticas o aspectos de carácter social.

\section{Conclusiones}

a. Existe evidencia de que la relación existente entre la prima por riesgo en Colombia para la última década es no contemporánea con el consumo privado, pero sí lo es con respecto a la tasa de desempleo. Adicionalmente, se encuentra que la prima por riesgo no cambia por lo menos para dos trimestres contiguos y que su comportamiento está permeado por eventos externos al nivel de actividad económica nacional acaecidos en periodos pasados muy cercanos.

b. Es necesario contar con más información para la estimación de la tasa libre de riesgo, la cual se espera llegue a medida que aumente la liquidez y variedad de títulos en los mercados de renta fija en Colombia. También, la juventud y alta concentración del mercado de renta variable hace más difícil el análisis estadístico del mercado colombiano e impide mejorar la robustez estadística de los modelos tal y como se logra en los referentes internacionales.

c. Finalmente, aun con las limitantes de información, el hallazgo estadístico para Colombia se suma a la evidencia encontrada en los referentes externos, en el sentido 
de que la prima por riesgo se comporta de forma contracíclica con respecto a la actividad económica, en este caso manifestada en las series de la tasa de desempleo y el consumo privado.

\section{Referencias}

Damodaran, A. (2008). What is the Riskfree Rate? A Search for the Basic Building Block. Recuperado de la base de datos SSRN: http://dx.doi.org/10.2139/ssrn.1317436

Damodaran, A. (2012). Equity Risk Premiums (ERP): Determinants, Estimation and Implications - The 2012 Edition. Recuperado de la base de datos SSRN: http://dx.doi. org/10.2139/ssrn.2027211

Fama, E. French, K. (1989). Business conditions and expected returns on stocks and bonds. Journal of Financial Economics, 25(1), 23-49. Recuperado de la base de datos Science Direct.

FAMA (2004). The Capital Asset Pricing Model: Theory and Evidence. Journal of Economic Perspectives, 18(3), 25-46. Recuperado de la base de datos JSTOR

Fernández, P. (2009). Prima de riesgo del mercado: histórica, esperada, exigida e implícita. Universia Business Review, 21, 56-65. Recuperado de http://ubr.universia.net/ pdfs_web/UBR001200956.pdf

Fernández, P. (2009). The Equity Premium in 100 Textbooks. Recuperado de la base de datos SSRN: http://dx.doi.org/10.2139/ssrn.1148373

FERSON, E. y HARVEY, C. (1991). The variation of economic risk premiums. Journal of Political Economy, 99(2), 385-415. Recuperado de la base de datos JSTOR

García, C. y Moreno, J. (2011). Optimización de portafolios de pensiones en Colombia: el esquema de multifondos, 2003-2010. Ecos de Economía, 15(33), 139-183. Recuperado de: http://publicaciones.eafit.edu.co/index.php/ecos-economia/article/ view/ 482/447

Hess, G. E IwATA, S. (1997). Measuring and Comparing Business - Cycle Features. Journal of Business and Economic Statistics, 15(4), 432-444. Recuperado de la base de datos JSTOR. 
Hodrick, R. y Prescott, E. (1997). Postwar U.S. business cycles: an empirical investigation. Journal of Money, Credit and Banking, 29(1), 1-16. Recuperado de la base de datos JSTOR.

Ibbotson, R. G. y Goetzmann, W. N. (2005). History and the Equity Risk Premium. Yale ICF Working Paper No. 05-04. Recuperado de la base de datos SSRN: http://ssrn. com/ abstract $=702341$

JARA, D. (2006). Propuestas dirigidas a mejorar la eficiencia de los fondos de pensiones. Borradores de Economía, 423, 1-14. Recuperado de http://www.banrep.gov.co/cgibin/ borradores/busqueda.pl

Kuznets, S. (1930). Equilibrium Economics and Business - Cycle Theory. The Quarterly Journal of Economics, 44(1), 381-415. Recuperado de la base de datos JSTOR.

Lettau, M.; Ludvigson, S. y Wachter, J. (2008). The Declining Equity Risk Premium: What role does macroeconomic risk play? Review of Financial Studies, 21(4), 1653-1687. Recuperado de la base de datos JSTOR

LucAs, R. (1978). Asset Prices in an Exchange Economy. Econometrica, 46(6), 14291445. Recuperado de la base de datos JSTOR

Lustig, H. y Verdelhan, A. (2012). Business cycle variation in the risk - return trade off. Journal of Monetary Economics, 59, 535-549. Recuperado de la base de datos ScienceDirect

Martínez, O. y Murcia, A. (2007). Desempeño financiero de los fondos de pensiones obligatorias en Colombia. Reporte de Estabilidad Financiera, 75-90. Recuperado de http://www.banrep.gov.co/publicaciones/pub_es_fin.htm\#2007

MARkowitz, H. (1952). Portfolio Selection. The Journal of Finance, 7(1), 77-91. Recuperado de la base de datos JSTOR

Menra, R. (2003). The equity premium: Why is it a puzzle? Financial Analysts Journal, 59(1), 54-69. Recuperado de la base de datos JSTOR

MıLL, J. S. (1848). Principles of Political Economy with some of their Applications to Social Philosophy. Recuperado de: http://www.econlib.org/library/Mill/mIP.html

Salomons, R. y Grootveld, H. (2003). The equity risk premium: emerging vs. developed markets. Emerging Markets Review, 4(2), 121-144. Recuperado de la base de datos ScienceDirect. 
Ciclo económico y prima por riesgo en el mercado accionario colombiano

ANDRÉS MAURICIO GÓMEZ SÁNCHEZ

JOSÉ GABRIEL ASTAIZA GÓMEZ

\section{Anexo}

Estimación de los modelos 1 y 4

\begin{tabular}{|c|c|c|c|c|c|}
\hline & $\begin{array}{l}\text { Dependent Variable: } \\
\text { Method: Least Squa } \\
\text { Date: 03/10/13 Tim } \\
\text { Sample (adjusted): } 2 \\
\text { Included observatio } \\
\text { Convergence achiev } \\
\text { Newey-West HAC Sta } \\
\text { Backcast: 2001Q4 }\end{array}$ & $\begin{array}{l}\text { RP } \\
\text { 0:59 } \\
\text { 2Q1 2012Q3 } \\
43 \text { after adjustr } \\
\text { after } 39 \text { iteratio } \\
\text { ard Errors \& Co }\end{array}$ & $\begin{array}{l}\text { hents } \\
\text { is } \\
\text { ariance (lag trun }\end{array}$ & cation = 3) & \\
\hline & Variable & Coefficient & Std. Error & t-Statistic & Prob. \\
\hline & C & -0.00117501 & 0.01299677 & -0.09040772 & 0.92845088 \\
\hline delo 1 & $\mathrm{CCP}$ & -0.18285786 & 0.21747338 & -0.84082871 & 0.40584516 \\
\hline & DES & 0.0325634 & 0.0427983 & 0.76085749 & 0.45156416 \\
\hline & CPIBC & -0.18968927 & 0.21815151 & -0.86952994 & 0.39016338 \\
\hline & $\mathrm{AR}(1)$ & 0.24423139 & 0.15450972 & 1.58068619 & 0.1224618 \\
\hline & $\mathrm{MA}(1)$ & -0.96846477 & 0.04246885 & -22.80412 & $2.20 \mathrm{E}-23$ \\
\hline & R-squared & 0.42786793 & Mean depende & nt var & -0.00209325 \\
\hline & Adjusted R-squared & 0.35055279 & S.D. dependent & t var & 1.01173719 \\
\hline & S.E. of regression & 0.81534168 & Akaike info crit & erion & 2.55836859 \\
\hline & Sum squared resid & 24.5969358 & Schwarz criteri & & 2.80411745 \\
\hline & Log likelihood & -49.0049248 & F-staticstic & & 5.53407661 \\
\hline & Durbin-Watson stat & 1.93168504 & Prob (F-staticst & & 0.00066692 \\
\hline & $\begin{array}{l}\text { Dependent Variable: } \\
\text { Method: Least Squa } \\
\text { Date: 03/06/13 Tim } \\
\text { Sample (adjusted): } \\
\text { Included observatio } \\
\text { Convergence achiev } \\
\text { Newey-West HAC Sta }\end{array}$ & $\begin{array}{l}\text { RP } \\
\text { 8:19 } \\
\text { 2Q2 2012Q3 } \\
\text { 42 after adjustn } \\
\text { after 65 iteratio } \\
\text { ard Errors \& Co }\end{array}$ & $\begin{array}{l}\text { ents } \\
\text { as } \\
\text { ariance (lag trun }\end{array}$ & cation = 3) & \\
\hline & Variable & Coefficient & Std. Error & t-Statistic & Prob. \\
\hline Modelo 4 & $\operatorname{CCP}(-1)$ & -0.050137 & 0.008308 & -6.034995 & 0.0000 \\
\hline & DES & 0.008358 & 0.003501 & 2.38734 & 0.0221 \\
\hline & $\mathrm{AR}(1)$ & 0.336257 & 0.194606 & 1.727892 & 0.0921 \\
\hline & $\mathrm{MA}(1)$ & -1.272209 & 0.400363 & -3.177635 & 0.0029 \\
\hline & R-squared & 0.591147 & Mean depende & nt var & 0.00221 \\
\hline & Adjusted R-squared & 0.558869 & S.D. dependent & t var & 0.140844 \\
\hline & S.E. of regression & 0.093545 & Akaike info crit & erion & -1.810351 \\
\hline & Sum squared resid & 0.332527 & Schwarz criteri & & -1.644859 \\
\hline & Log likelihood & 42.01737 & F-staticstic & & 2.244009 \\
\hline
\end{tabular}

